\title{
$S^{5}(1)$ 中极小超曲面的几何与拓扑障碍
}

\author{
林峻崌夏昌玉
}

(复旦大学数学研究所, 上海)

\section{一、引 高}

极小子流形理论中一个基本问题是：对充分大的 $N$,一个紧流形能否极小蚮入到 $S^{N}(1)$ 中 ${ }^{[1]}$. 本文得到一个 4 维流形能作为 $S^{5}(1)$ 中极小超曲面的拓扑障碍. 即有下面的

\section{定理 1 如果 $M^{4} \longrightarrow S^{5}(1)$ 为极小浸入, 则 $\left|W^{+}\right|-\left|W^{-}\right|$.}

作为定理 1 的推论, 我们知道: 如果 $M^{4} \longrightarrow S^{5}$ (1) 为一极小浸人, 则 $M$ 的差号为零. 据 此, $C P^{2}$ 和 $K_{3}$ 不是 $S^{3}(1)$ 中的极小超曲面且 $S^{3}(1)$ 中的所有极小超曲面可以分解成 $C P^{2}, K_{3}$, $S^{4}$ 和 $S^{2} \times S^{2}$ 的连通和.

众所周知, 在极小子流形理论中, Simons 不等式可以被用来得到 $s$ 的 Pinching 定理. 如: 当 $S<n(2-1 / P)$ 时, $M$ 是全测地的 ${ }^{[2]}$. 在本文中, 通过使用 Gauss-Bonnet 定理, 我们 得到了下面的整体 Pinching 定理.

定理 2 设 $M^{4} \longrightarrow S^{5}(1)$ 为一定向极小超曲面, $\chi \leqslant 2$, 则存在一个万有常数 $c>0$, 使得, 当 $\int_{M} S^{2}<c$ 时, $M$ 是全测地的.

定理 2 是由下面的定理得到的.

定理 3 设 $M^{4} \longrightarrow S^{5}(1)$ 为 $S^{5}$ 中的定向极小超曲面, 如果 $M$ 非全测地, 则存在万有常 数 $c>4 \cdot 10^{-33}$ 使得

$$
\int_{M} S^{2} \geqslant 64 \pi^{2}(2-\chi) / 3+c .
$$

对单位球面中的极小曲面, 我们也有下面的

定理 4 设 $M^{2} \longrightarrow S^{n}(1)$ 为定向的非全测地亏格为 $g$ 的极小曲面. 则存在 常数 $c>$ $(4 n-10) /\left(7+2 e^{4 c}\right)$ 使得

$$
\begin{aligned}
& \int_{M} S \geqslant 8 \pi g+c . \\
& =\text { 、预 备 知 识 }
\end{aligned}
$$

设 $M^{n}$ 为 $n$ 维 Riemann 流形. 设 $R_{i j k l}, R_{i i}$ 和 $\rho$ 分别为 $M$ 在某一正交标架下的 Riemann, Ricci 曲率张量和纯量曲率 $M$ 的 Weyl 共形曲率张量和 Einstein 曲率张早, 分别由下面的式 子定义

$$
W_{i j k l}=R_{i j k l}-\frac{1}{2}\left(R_{i k} \delta_{i l}-R_{i l} \delta_{i k}+R_{i l} \delta_{i k}-R_{j k} \delta_{i l}\right)
$$

本文 1987 年 5 月4 日收到. 


$$
\begin{aligned}
& +\rho / 6\left(\delta_{i l} \delta_{i k}-\delta_{i l} \delta_{i k}\right), \\
& E_{i j}=R_{i i}-\rho / n \delta_{i i} .
\end{aligned}
$$

设 $M^{4}$ 为定向 4 维流形,其 Euler 示性数、差号、曲率张量和 Einstein 张量分别为 $\chi, \sigma$, $R$ 和 $E . d x^{2} d x^{2} d x^{3} d x^{4}$ 为 $M$ 的定向体积元, 由下式定义 $W_{i j k l}^{+}, W_{i j k l},\left|W^{+}\right|^{2}$ 和 $\left|W^{-}\right|^{2}$ :

$$
\begin{gathered}
W_{i ; k l}^{+} d x^{k} \wedge d x^{l}=\frac{1}{2} W_{i j k l}\left(d x^{k} \wedge d x^{l}+{ }^{*} d x^{k} \wedge d x^{l}\right), \\
W_{i j k l} d x^{k} \wedge d x^{l}-\frac{1}{2} W_{i j k l}\left(d x^{k} \Lambda d x^{l}-{ }^{*} d x^{k} \Lambda d x^{l}\right), \\
\left|W^{+}\right|^{2}=\sum W_{i ; k l}^{+} W_{i ; k l}^{+} \quad\left|W^{-}\right|^{2}=\sum W_{i ; k l}^{-} W_{i ; k l}^{-} .
\end{gathered}
$$

引理 1 (4 维 Gauss-Bonnet 公式 ${ }^{[3]}$ )

$$
\int_{M}|R|^{2}=4 \int_{M}|E|^{2}+32 x^{2} x \text {. }
$$

引理 2 (Riemann-Roch-Hirzebruch 定理 ${ }^{(4)}$ )

$$
12 \pi^{2} \sigma=\int_{M}\left(\left|W^{+}\right|^{2}-\left|W^{-}\right|^{2}\right) .
$$

引理 $3^{[j]}$ 设 $M^{n} \longrightarrow S^{*+p}(1)$ 为紧极小子流形. 如果 $M^{n}$ 非全测地, 则存在万有常数 $\therefore(n)>0$, 使得

$$
V(M)>(1+c(n)) V\left(S^{n}(1)\right) \text {. }
$$

\section{三、定理 1 的证明}

由定义,我们有

$$
\left|W^{+}\right|^{2}-\sum_{i, j}\left(\left|W_{i j 12}+W_{i j 34}\right|^{2}+\left|W_{i j 13}-W_{i j 24}\right|^{2}+\left|W_{i j 4}+W_{i j 23}\right|^{2}\right) .
$$

由于 $\left(h_{i j}\right)$ 对称,我们可选取 $M$ 在一点的正交基,使得

$$
\begin{aligned}
& h_{i j}=\lambda_{i} \delta_{i i} \text {, } \\
& R_{i j}=\left(3-\lambda_{i} \lambda_{j}\right) \delta_{i j} \text {, } \\
& \rho=12-S \text {, } \\
& W_{i j 1}+W_{i j 4}=R_{i j 12}+R_{i j 4}-\frac{1}{2}\left(R_{i 1} \delta_{i 2}-R_{i 2} \delta_{j 1}\right. \\
& \left.+R_{j 2} \delta_{i 1}-R_{j i} \delta_{i 2}+R_{i j} \delta_{j 4}-R_{i j} \delta_{j 3}+R_{j 4} \delta_{i 3}-R_{i 3} \delta_{j 4}\right) \\
& +\rho / 6\left(\delta_{i 1} \delta_{i 2}-\delta_{i 2} \delta_{j 1}+\delta_{i 3} \delta_{i 4}-\delta_{i 4} \delta_{i 3}\right) \\
& -\left(\left(\lambda_{i}+\lambda_{i}\right)^{2} / 2-S / 6\right)\left(\delta_{i 1} \delta_{i 2}-\delta_{i 2} \delta_{j 1}+\delta_{i 3} \delta_{i 4}-\delta_{i 4} \delta_{i 3}\right) \text {, } \\
& \sum_{i, j}\left|W_{i j 12}+W_{i j 34}\right|^{2}=\frac{1}{2}\left(\left(\left(\lambda_{1}+\lambda_{3}\right)^{2}-S / 3\right)^{2}+\left(\left(\lambda_{3}+\lambda_{4}\right)^{2}-S / 3\right)^{2}\right) \text {, } \\
& \left.\sum_{i, i}\left|W_{i j \mid 3}-W_{i j 34}\right|^{2}-\frac{1}{2}\left(\left(\left(\lambda_{1}+\lambda_{3}\right)^{2}-S / 3\right)^{2}+\left(\lambda_{2}+\lambda_{4}\right)^{2}-S / 3\right)^{2}\right), \\
& \sum_{i, j}\left|W_{i j 4}-W_{i j 33}\right|^{2}=\frac{1}{2}\left(\left(\left(\lambda_{1}+\lambda_{4}\right)^{2}-S / 3\right)^{2}+\left(\left(\lambda_{2}+\lambda_{3}\right)^{2}-S / 3\right)^{2}\right) . \\
& \text { 由(11)，(16)，(17)，(18)式, Gauss 方程, } \Sigma \lambda_{i}=0 \text { 及 } \sum \lambda_{i}^{2}=S \text {, 有 } \\
& \left|W^{+}\right|^{2}=7 S^{2} / 6-2 \sum \lambda_{k}^{4} \text {. }
\end{aligned}
$$

类似的计算表明: $\left|W^{-}\right|^{2}-\left|W^{+}\right|^{2}$. 


\section{由定理 1 及引理 2, 我们有下面的}

推论 1 如果 $M^{4} \longrightarrow S^{3}(1)$ 为极小漫人, 则 $M$ 的差号为零.

注. Bryant 曾证明 ${ }^{(0)}$, 每一紧 Riemann 面可以共形极小浸人到 $S^{4}$ 中, 定理 1 说明类似 的关于 $M^{4}$ 的结果将不成立. 即, 如果预先给定 $M^{4}$ 上的共形结构, 一般地不可能有此共形结 构中的 Riemann 度量,使得此 Riemann 流形可极小漫人到 $S^{3}(1)$ 中. 因为在此 $W^{+}, W^{-}$均 为共形不变量.

\section{四、定理 3 和定理 4 的证明}

定理 3 的证明 由(8),(12)式和 Gauss 方程,我们有

$$
\int_{M}\left(3 S^{2} / 2+12-2 S\right)=16 \pi^{2} \chi+3 \int_{M} \sum_{k} 2 k^{4} \text {. }
$$

由 $\left|W^{+}\right|^{2}$ 的计算, 我们知 $\sum_{k} \lambda_{k}^{4} \leqslant 7 S^{2} / 12$, 这样, 由 Simons 不等式 $\int_{\boldsymbol{u}} 4 S \leqslant \int_{\boldsymbol{u}} S$, 和(20)式,我们有

$$
\int_{M} S^{2} \geqslant 64 \pi^{2}(2-\chi) / 3+c,
$$

经过准确的计算知, $c>48 \pi^{2} /\left(11+2 e^{20}\right) \doteq 4 \cdot 10^{-33}$.

定理 4 的证明设 $K$ 为 $M^{2}$ 的 Gauss 曲率, 则由 Gauss 方程和引理 3, 我们有

$$
\int_{M} S=2 V(M)-8 \pi(1-g) \geqslant 8 \pi g+c \text {. }
$$

推论 2 设 $M^{2} \longrightarrow S^{n}(1)$ 为亏格为 8 的定向极小曲面, 则存在万有学数 $c>0$, 使当 $\int_{M} S<8 \pi g+c$ 时, $M$ 全测地.

引理 4 设 $M^{2} \longrightarrow S^{n+p}(1)$ 为闭极小子流形, 则任给 $t \geqslant 0$,

$$
\int_{M} S^{t+1}(S-n /(2-1 / p)) \geqslant 0 .
$$

证由文献[2],我们有

$$
\frac{1}{2} \Delta S \geqslant S(n-(2-1 / p) S),
$$

任给 $\varepsilon>0$, 我们有

$$
\begin{gathered}
1 / 2 \Delta S(S+\varepsilon)^{t} \geqslant S(n-(2-1 / p) S)(S+\varepsilon)^{t}, \\
\Delta(S+\varepsilon)^{t+1}=(t+1) t(S+8)^{t-1}|\nabla S|^{2}+(t+1)(S+\varepsilon)^{t} \Delta S .
\end{gathered}
$$

由(25), (26)式, Green's 公式及假设 $\iota \geqslant 0$, 我们有

$$
\begin{aligned}
& \int_{M} S(n-(2-1 / p) S)(S+\varepsilon)^{z} \leqslant \frac{1}{2} \int_{M} \Delta S(S+8)^{\varepsilon} \\
= & \int_{M}(S+\varepsilon)^{t+1} / 2(t+1)-\frac{t(t+1)}{2}(S+8)^{t-1}|\nabla S|^{2} \\
\leqslant & 0 .
\end{aligned}
$$

在 (27)式中令 $B \rightarrow 0$, 即得引理 4 .

推论 3 设 $M^{4} \longrightarrow S^{5}(1)$ 为闭定向极小超曲面, $\chi \leqslant 2$. 如 $S \not \equiv 0$, 则存在万有常数 $c>0$ 使得对任意整数 $p \geqslant 2$, 有 


$$
\int_{M} S^{p} \geqslant 4^{p-1} \cdot c .
$$

推论 4 设 $M^{2} \longrightarrow S^{n}(1)$ 为亏格为 $g$ 的闭定向极小曲面. 如 $S \neq 0$, 则存在常数 $c>0$ 使得任给整数 $p \geqslant 1$, 我们有

$$
\int_{M} S^{p} \geqslant((2 n-4) /(2 n-5))^{p-1}(c+8 \times 8) .
$$

注. 最近, 我们已经证明关于球面中的极小子流形也有类似的 Pinching 定理, 详细情况 将另文讨论.

\section{* 苦 文}

[1] Yau, S. T., Problem, section, in Seminar on Differendial Geometry (Ed. S. T. Yau), Princevon Uaiv. Pren, 1982.

[2] Simons, J., Ann. of Math., 88(1968), 62.

[3] Willmore, T. J., Total Curvature in Riemannian Geometry, Ellis, 1982, 80.

[4] Poon, S. Y, J. Diff. Geom, 24(1986), 97.

[5] Cheng, S. Y., Li, P. \& Yau, S. T., Amer. J. Mask, 106 (1984), 1033.

[6] Bryant, R. L, J. Diff. Geom, 17(1982), 455. 